\title{
Plant Size and Number Affect Genetic Analysis and the Improvement of Fruit and Nut Tree Cultivars
}

\author{
Paul E. Hansche \\ Department of Pomology, University of California, Davis, CA 95616
}

\begin{abstract}
Dwarfing genes are among the most widely known plant mutations (Pleton, 1964), which suggests that a reasonable search, even in the absence of mutagenic agents, would likely turn up genetic dwarfs in the tree fruit and nut species where they have not yet been reported. Genetic dwarfs play a prominent role in numerous agronomic crops; e.g., sunflower (Allard, 1961), barley (Ali et al., 1978), wheat and rice (Dalrymple, 1980), sorghum (Schertz et al., 1974), tomato (Stevenson and Tomes, 1958), cucurbits (Mohr, 1962), peas (Macomb and Macomb, 1970), and currents and gooseberries (Bauer, 1957). Trees of Prunus persica L. Batsch and $P$. amygdalus L. Batsch have been genetically reduced in size by $30 \%$ to $90 \%$, apparently without any serious adverse side effects (Hooper, 1867; Lammerts, 1945; Hansche et al., 1985; Hansche, 1989, unpublished data). All of this information suggests that fruit and nut trees can readily be reduced in size via single mutant recessive genes.
\end{abstract}

Obviously, small seedlings occupy less space than large ones, and consequently are less expensive to rear. This situation presents those interested in genetic analysis and manipulation of these species with the option of working with a relatively small number of large plants or a relatively large number of small plants.

The duration of juvenility in Prunus, Malus, and Pyrus also has been shown to be readily amenable to genetic manipulation (Hansche, 1986; Visser, 1965,1972, 1976; Zielinski, 1963) and thus provides an even greater range of options to those interested in genetic analysis and fruit and nut tree cultivar improvment; extending from very large plants with very long sexual cycles to small plants with short sexual cycles. The relative merits of these options are addressed here.

\section{The role of plant size in genetic analysis}

The genetic analyses and manipulations referred to here are those that depend on sexual reproduction and on observation of the phenotypic consequences of the meiotic process among segregating progeny. They include the implementation of recombinant DNA technologies [such as DNA restriction fragment length polymorphism (RFLP) map-

Received for publication 11 May 1989; The cost of publishing this paper was defrayed in part by the payment of page charges. Under postal regulations, this paper therefore must be hereby marked advertisement solely to indicate this fact. ping] as breeding tools, as well as the classic Mendelian and quantitative genetic methodologies.

Usually, specific genotypes can be expected to arise within segregating progenies at a frequency predicted by the independent transmission of alleles of each parental gene involved. (Of course, in unusual cases where genetic linkage plays a significant role, specific genotypes could be expected to arise more or less frequently.) The relationship between population size and the probability of a seedling arising that is homozygous for recessive alleles of three to five independent genes in this population is illustrated in Fig. 1 .

The probability of a seedling simultaneously expressing values in the upper $10 \%$ of the $\mathrm{F}_{2}$ distribution for three to five independently segregating quantitative traits is equally well-illustrated in Fig. 1; e.g., fruit size, color, flavor, disease resistance, and productivity. Clearly, the number of seedlings that comprise fruit and nut tree breeding stocks not only imposes limits on the resolution of features of the genetic mechanism that depend on specific allelic combinations at more than a very few gene loci, it also limits the likelihood of any seedling simultaneously expressing uncommonly high values for only a very small number of traits. The relationships depicted in this figure point directly to the smallest plant size as the most desirable option for geneticists, and other re-

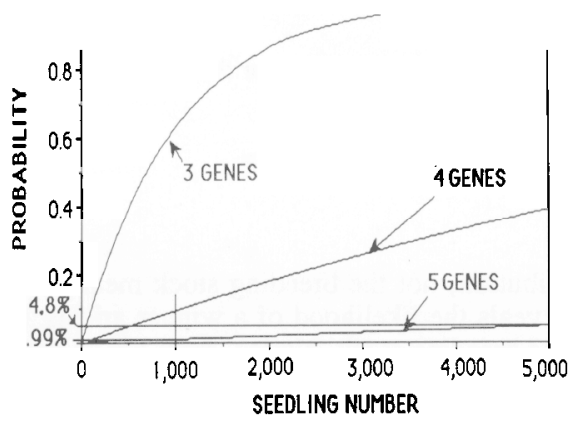

Fig. 1. The probability of at least one seedling that is homozygous for recessive alleles of three to five independent genes arising among the progeny of a random mating $\mathrm{F}_{1}$ population. The frequency of recessive alleles is assumed to be 0.33 . Note the probability of at least one seedling being homozygous for all five recessive alleles is only $0.99 \%$ in breeding stocks comprising 1000 seedlings, and $4.8 \%$ in breeding stocks comprising 5000 seedlings. searchers, because small plants facilitate use of many seedlings.

The role of plant size on the rate of genetic improvement

Response to selection: a) sexually reproduced crops. However small the likelihood is of a seedling arising that meets a given set of criteria in a breeding stock, it can be increased by genetically "improving" the breeding stock. This can be accomplished in genetically variable breeding stocks by appropriately selecting and mating parents. The distribution of values expressed by the offspring of selected parents can be expected to shift upward. Consequently, a greater proportion of individuals among their progeny will meet criteria based on high values.

A common approach to the improvement of breeding stocks is to select as parents those individuals that perform very well (e.g., that have the sweetest fruit, the highest blush, or that are most resistant to disease) and mate them randomly inter se. This strategy is generally referred to as mass selection (Allard, 1960). The selection intensity, $i$, can be defined in units of the standard deviation (SD), as the average amount $\left(\overline{\mathrm{X}}_{\mathrm{sp}}\right)$ by which the performance of selected parents surpasses the mean $\left(\overline{\mathrm{X}}_{\mathrm{bs}}\right)$ of the breeding stock, where $\mathrm{i}=$ $\left(\overline{\mathrm{X}}_{\mathrm{sp}}\right)-\left(\overline{\mathrm{X}}_{\mathrm{bs}}\right)$.

The effect of this procedure on the subsequent performance of breeding stock progeny of selected parents can be quantified (Fig. 2).

The amount (in units of the standard deviation) by which the selection increases the mean of the progeny is called the "response to selection", and is symbolized by R. The latter is linearly related to $\mathrm{i}$ by $\mathrm{h}^{2}$; i.e., $\mathrm{R}=$ $\mathrm{ih}^{2}$. In the plant and animal breeding literature, $\mathrm{h}^{2}$ is called the heritability of the trait (Falconer, 1981). It is that portion of the variation in the population attributable to the additive statistical effects of genes. The heritability, $\mathrm{h}^{2}$, tells us the degree to which we can predict mean offspring performance from that of their parents.

Numerous experiments in many crop species have shown that $\mathrm{R}$ (the amount that the mean performance of the breeding stock, $\overline{\mathrm{X}}_{\mathrm{bs}}$, increases in each subsequent cycle) will remain constant if the intensity of selection (i) is maintained at a constant value as this process is repeated (Fig. 3). This process resides at the core of cultivar improvement in many agronomic and vegetable crops that are normally sexually propagated and maintained (Allard, 1960). The mean perform- 


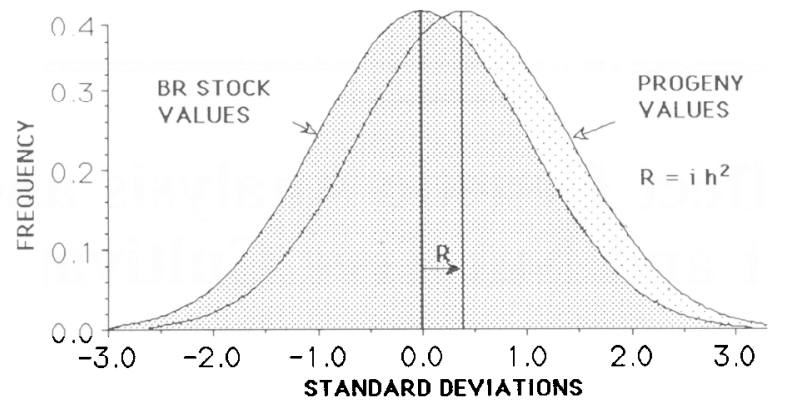

Fig. 2. Distribution of parental breeding stock values and those of progeny of selected parents, expressed in terms of standard deviations from the mean of the parental breeding stock.

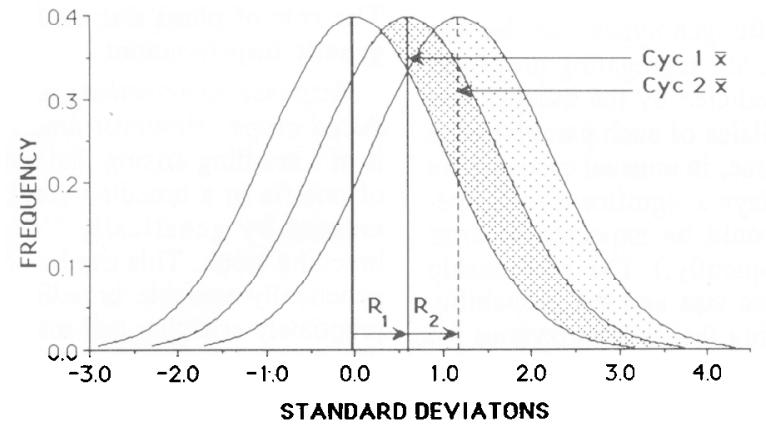

Fig. 3. The first and second generation responses, $R_{1}$ and $R_{2}$, of a hypothetical breeding stock, to two cycles of a constant selection intensity, $i$.

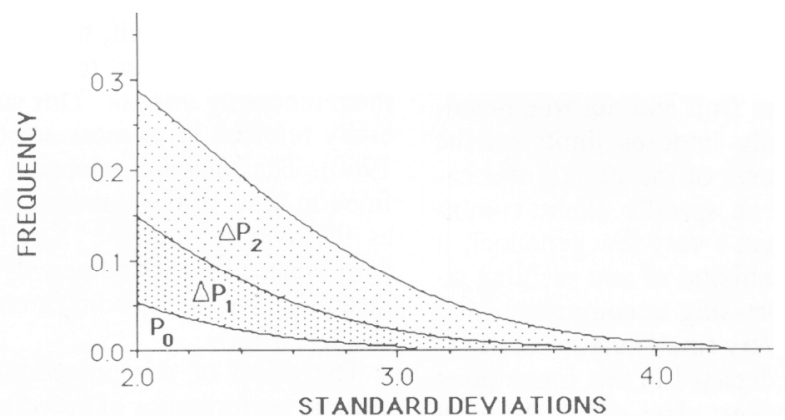

Fig. 4. The area under the upper tail of each of the three distributions represent the probability, $\mathrm{P}_{0}$, of a random seedling from the initial breeding stock, $X_{0}$, expressing a trait value 2 SDS above the mean of the original population: $P_{0}=P\left[X_{0}>2 \mathrm{SD}_{0}\right]=\int_{2 s i d}^{-} \mathrm{N}\left(\mathrm{O}, \sigma^{2}\right) \mathrm{dx}$; the probability after one

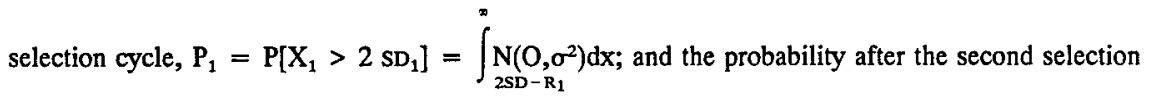
cycle, $\mathrm{P}_{2}=\mathrm{P}\left[\mathrm{X}_{2}>2 \mathrm{SD}_{2}\right]=\int_{2 \mathrm{SD}-\mathrm{R}_{1}-\mathrm{R}_{2}}^{\mathrm{N}\left(\mathrm{O}, \sigma^{2}\right) \mathrm{dx}} . \Delta \mathrm{P}_{1}=\mathrm{P}_{1}-\mathrm{P}_{0}, \Delta \mathrm{P}_{2}=\mathrm{P}_{2}-\mathrm{P}_{1}$.

ante of cultivars generated in this manner generally reflects the mean performance of the breeding stock.

Response to selection: $b$ ) asexually reproduced crops. In crops that are normally asexually propagated, the best seedling (the winner) is picked from the uppermost end of the distribution of seedling performances, cloned, and released as a cultivar. Consequently, the breeder is less interested in increasing the mean of the breeding stock than in increasing the probability of a winner arising in the breeding stock, $\mathrm{P}[\mathrm{w}]$. It is the area under the curve at the upper end of the dis- tribution, not the breeding stock mean, that reveals the likelihood of a winner arising in the breeding stock. Notice, from Fig. 3, that the area under the curve at the upper end (for example, the area exceeding the mean of the original breeding stock by 2 SDS) increases as the mean of the breeding stock moves to the right on the scale in response to selection. The upper ends of the distributions depicted in Fig. 3 are enlarged in Fig. 4. Notice also that, whereas the rate of response of the mean is constant(i.e., $R_{1}=R_{2}$ in Fig. 3), the rate of increase in the area under the upper end of the breeding stock distribution increases each cycle; i.e., $\Delta \mathrm{P}_{2}=\left(\mathrm{P}_{2}-\mathrm{P}_{1}\right)$ $>\Delta \mathrm{P}_{1}=\left(\mathrm{P}_{1}-\mathrm{P}_{0}\right)$. The increases in $\mathrm{P}_{\mathrm{i}}$ and $\Delta \mathrm{P}_{\mathrm{i}}$, from cycle to cycle, are of great relevance to cultivar improvement in asexually reproduced cultivars.

The effect of seedling number on response to selection. The rate, $\mathrm{R}$, at which selection increases the mean of breeding stocks is influenced by the number of seedlings therein. The rate, $\Delta \mathrm{P}$, at which selection increases the frequency of seedlings that meet selection criteria, and $\mathrm{P}[\mathrm{w}]$, the likelihood of a winner arising in the breeding stock, are highly sensitive to population size. In fact, the number of seedlings comprising the breeding stock dramatically affects $\Delta \mathrm{P}$ and $\mathrm{P}[\mathrm{w}]$ in situations that pertain widely in tree fruit and nut cultivar improvement programs; i.e., where selection is practiced on numerous traits in populations that comprise a few thousand seedlings.

The relationship can be illustrated by comparing the effects of one selection cycle on $\mathrm{R}, \Delta \mathrm{P}$, and $\mathrm{P}[\mathrm{w}]$ in two hypothetical breeding stocks; one comprising 1000 and the other 5000 seedlings. [The population size difference here was chosen to reflect the fact that five dwarf peach or almond seedlings can readily be reared in the space required by one standard peach seedling (Hansche et al., 1985).] Assume the objective is a seedling (a winner) that performs in the upper $10 \%$ of the two hypothetical breeding stock distributions for each of five independently inherited traits. This goal implies that "the object seedling" must express values for all five traits that exceed the mean of the two hypothetical breeding stocks by 1.28 SDS of the mean of the original breeding stock $\left(\mathrm{SD}_{\mathrm{o}}\right)$. Assuming these traits are transmitted independently of one another, the expected frequency of seedlings meeting all five criteria would be only $0.10^{5}$, or $1 / 100,000$. Consequently, a seedling meeting all of these criteria (a winner) would not likely be present in either hypothetical breeding stock. These populations are simply too small (see Fig. 1). Furthermore, assume mass selection is practiced in these populations to increase the probability that a seedling meeting all five criteria will arise among the progeny of selected parents; i.e., assume selection is practiced to increase $\mathrm{P}[\mathrm{w}]$. The intensity of selection that can be applied to each of these traits is limited by the number of seedlings in the hypothetical breeding stocks. For example, the relatively mild requirement that parents perform only in the upper $38 \%$ of the two hypothetical populations with respect to all five traits would be expected to rule out 992 of the 1000 seedlings comprising one breeding stock, and 4960 of the other. Thus, $\mathrm{i}=1$, due to this mild requirement. This result derives from the joint probability of performance in the upper $38 \%$ of the distribution for all five traits; i.e., $0.38^{5}=$ 0.0079 or $8 / 1000$ or $40 / 5000$. Selection of this intensity is expected to lead to a response in both populations of $\mathrm{R}=\mathrm{ih}^{2}$, or since $i=1, R=h^{2}$ for each of the five traits.

To facilitate this analysis, the additional 
assumption is made that heritabilities of these traits are all equal to 0.3 [which is within the range of estimates of the heritabilities of such traits in our peach breeding stock (Hansche and Beres, 1980)]. This selection would shift the distribution of progeny values of each of the five traits upward by 0.3 SD of the mean measurement of each trait (Fig. 5). As a result, the expected proportion of progeny meeting each of the performance criteria. $\mathrm{P}_{1},=\mathrm{P}_{0}+\Delta \mathrm{P}$, would have increased from $10 \%$ to $15 \%$ (Fig. 5). This expectation is independent of the number of seedlings in the breeding stocks.

However, a comparison of responses resulting from selection of the same number of seedlings from each breeding stock reveals one of the advantages provided by the larger population. For example, the selection intensity in the 5000-seedling breeding stock would be 1.23 rather than 1 were the best eight seedlings, rather than the best $0.8 \%$ of the seedlings, chosen as parents. With this strategy, the parents in the 5000-seedling breeding stock would be chosen from the upper $27 \%$ (since $0.27^{5} \cong 8 / 5000$ ), rather than the upper $38 \%$, as they were in the 1000 seedling breeding stock (since $0.38^{5} \cong 8$ / 1000). The differences in selection intensity and in percentage seedlings selected when the best eight seedlings in each stock are used as parents are illustrated in Fig. 6.

The mean response for each of the five traits in the population comprising 5000 seedlings would directly reflect the $23 \%$ increase in the selection intensity; i.e., where $0.8 \%$ of the seedlings (i.e., 40) were selected as parents, $\mathrm{i}=1$ and $\mathrm{R}=0.30 \mathrm{sD}$; where eight seedlings were selected as parents, $\mathrm{i}=1.23$ and $\mathrm{R}=0.369 \mathrm{SD}$. However. as a result of the $23 \%$ increase in the progeny mean, the proportion of seedlings exceeding a value of 1.28 SD above the original breeding stock mean would have increased by $60 \%$, rather than only $23 \%$; i.e., for $\mathrm{i}=1.23$, $\Delta \mathrm{P}[1.23]=8 \%$, but for $\mathrm{i}=1, \Delta \mathrm{P}[1]=$ $5 \%$ (Fig. 7). This increase is of great relevance to breeders of asexually reproduced cultivars.

The $23 \%$ increase in $\mathrm{i}$ and in $\mathrm{R}$ that generated the $60 \%$ increase in $\Delta \mathrm{P}[1.23]$ also increases the probability of at least one seedling in the breeding stock meeting all five criteria, $\mathrm{P}[\mathrm{w}]$. The probability of a winner having arisen in the original breeding stock, comprising 1000 seedlings, would be $\mathrm{P}[\mathrm{w}]_{1000}$ $=1-\left(1-0.10^{5}\right)^{1000}=0.0099$. In the population of 5000, its expectation would be $\mathrm{P}[\mathrm{w}]_{5000}=1-\left(1-0.10^{5}\right)^{5000}=0.048$. The expectation, $\mathrm{P}[\mathrm{w}]$, of at least one such seedling arising among the progeny of the two populations subjected to a selection intensity of $\mathrm{i}=1$ wouldbe: $\mathrm{P}[\mathrm{w}]_{1000}=1-$ $\left(1-0.15^{5}\right)^{1000}=7 \%$, and $\mathrm{P}[\mathrm{w}]_{5000}=1-$ $\left(1-0.15^{5}\right)^{5000}=29 \%$. However, the expectation in the population of 5000 subjected to the more intense selection, where $\mathrm{i}=$ 1.23 , would be $\mathrm{P}[\mathrm{w}]_{5000}=1-\left(1-18^{5}\right)^{5000}$ $=39 \%$ (Fig. 8 ).

The more than 5-fold advantage of the larger over the smaller population $\left(\mathrm{P}[\mathrm{w}]_{5000}\right.$ $=39 \%$ vs. $\mathrm{P}[\mathrm{w}]_{1000}=7 \%$ ) is due to:a) the

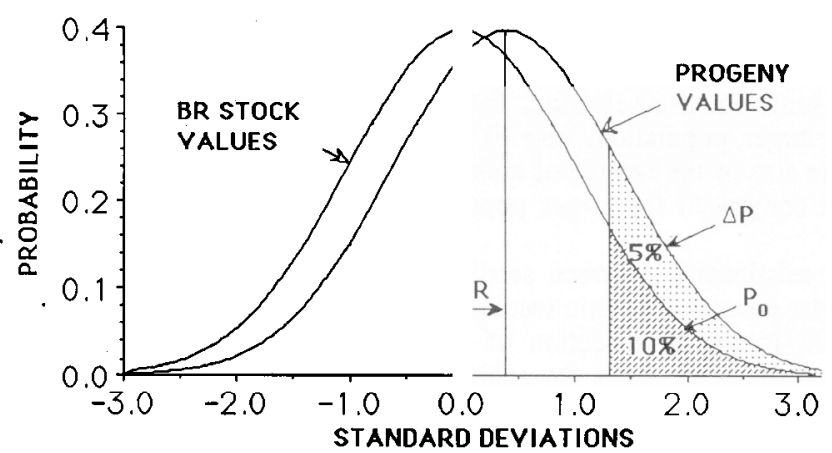

Fig. 5. Distribution of values expressed in the parental breeding stocks and among the progeny of parents subjected to a selection intensity $i=1$ for each of the five traits under selection. $h^{2}$ equals 0.3 for all five traits.

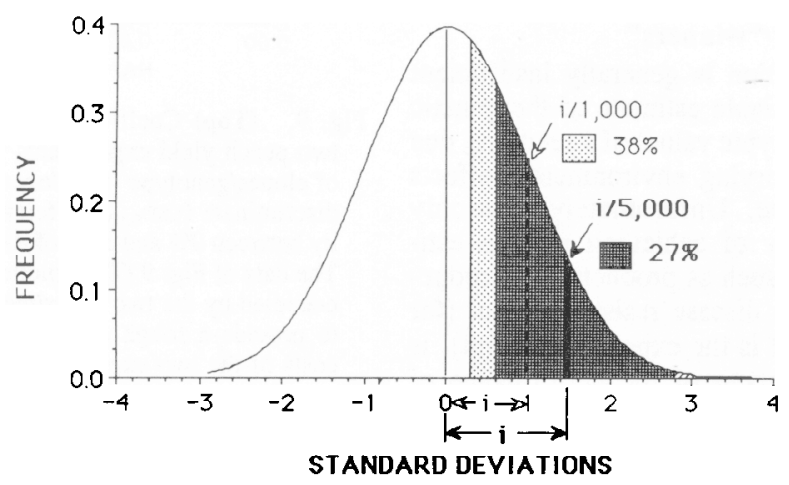

Fig. 6. Distribution of breeding stock values illustrating selection intensities, $i_{1000}=1$, and $i_{5000}=$ 1.23 , pertaining to each of the five traits, where $8 / 1000$ and $8 / 5000$ are selected as parents.

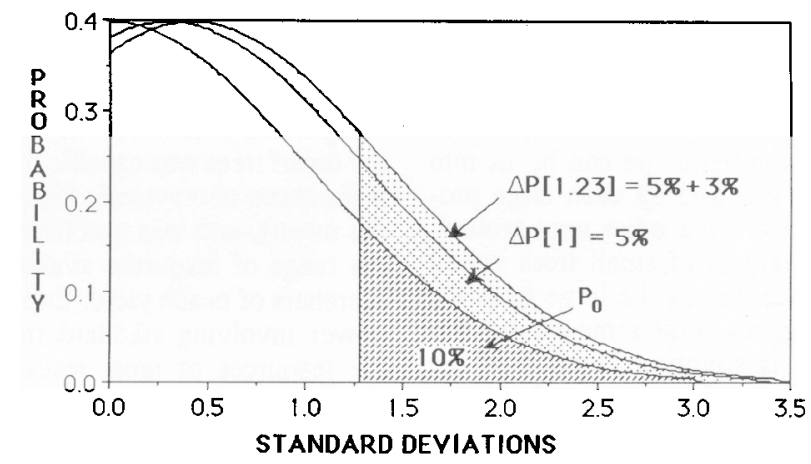

Fig. 7. The upper tail of the original breeding stock (圈) of the progeny derived from parents subjected to a selection intensity, $i=1$ (ㄱ) and of the progeny derived from the parents subjected to a selection intensity, $\mathrm{i}=1.23(0)$.

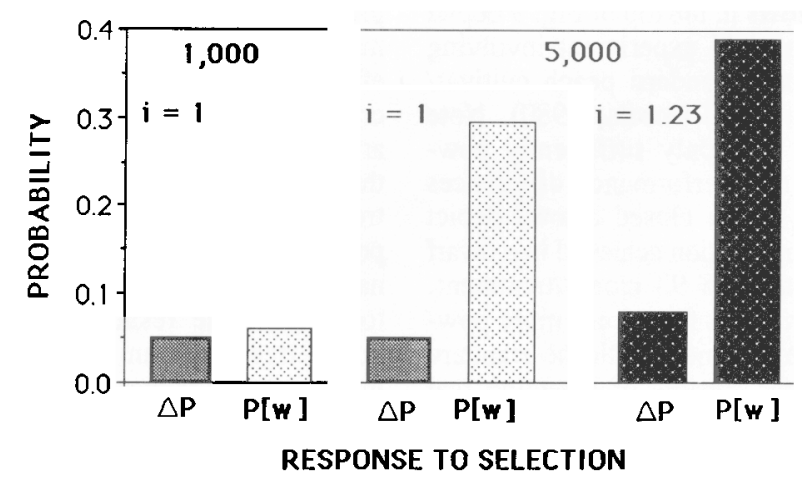

Fig. 8. Selection responses in populations comprising 1000 and 5000 seedlings. $\Delta P$ is the change in the frequency (probability) of seedlings that meet at least one of the five criteria. $\mathrm{P}[\mathrm{w}]$ is the probability of at least one "winner" arising among the progeny of selected parents; i.e., a scedling that meets all five criteria. 
increase in the frequency of desired genotypes among the meiotic products of parents subjected to more intense selection, facilitated by the larger population, and $b$ ) the increase in the size of the sample of meiotic products that comprised the larger population.

The direct relationship between seedling number and the power of genetic resolution was illustrated in the first section of the analysis. It can be seen, from the analysis presented in this section, that seedling number also has a less direct, but equally large, effect on the rate at which selection can increase the probability of desirable seedlings arising in breeding stocks.

\section{The effect of seedling number on discriminating "winners"}

One observation is generally insufficient to present a reliable estimate of the genetic potential (genotypic value) of a seedling, due to randomly varying environmental effects on performance. Unfortunately, the only practicable way of achieving reliable estimates of traits such as productivity, productive efficiency, disease resistance, etc. (for which the plant is the experimental unit), is through an average of the performance of replicate clones. The increased confidence achieved therefrom derives from the fact that the variance of measurement means, $\mathrm{V}_{\overline{\mathbf{x}}}$ equals $1 / n^{\text {th }}$ the variance of individual measurements; i.e., $V_{\bar{x}}=(1 / n) V i$, where $n$ is the number of measurements comprising the mean.

Real differences can be detected among genotypes of small propagules that are only $1 / \sqrt{ } \mathbf{n}$ the magnitude of those detectable among large propagules where $\mathrm{n}$ small propagules of a given genotype can be fit into the same space required by each large propagule. Or to put it the other way around, the space requirement of small trees would be only $1 / n$ times that of the large trees for detecting differences of the same magnitude. This difference is simply a function of the relationship between the variance of individual measurements and that of means (shown in the paragraph above). The relationship between number of replicate trees and the level of discrimination of genetic effects in two peach yield trials are depicted in the top of Fig. 9.

The open arrows in the top of Fig. 9 depict the results of a yield experiment involving 12 clones of each standard peach cultivar/ treatment (Beutel and Gerdts, 1980). Note the experiment was only sufficiently powerful to detect real performance differences $>33 \%\left(\mathrm{LSD}_{0.05}\right)$. The closed arrows depict the level of discrimination achieved in a dwarf peach yield trial with 93 clones/treatment. Note that it was about four times more powerful than the experiment with the standard trees; i.e., it was capable of detecting real differences in performance greater than $\approx 8 \%$. Data in the figure clearly illustrate the fact that more trees/treatment will generally lead to an increase in the power of experiments due to the fact that $V_{\overline{\mathbf{x}}}=(1 / \mathrm{n}) \mathrm{V}_{\mathrm{i}}($ see above $)$. [Estimated yield of the dwarf trees, $15.4 \mathrm{~kg}$ /
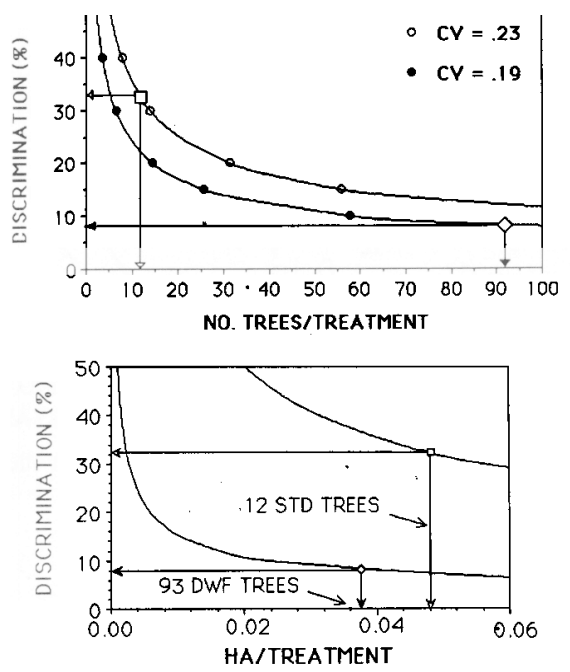

Fig. 9. (Top) Coefficients of variation (CV) of two peach yield experiments relate the number of clones/genotype (trees/treatment) required to discriminate $\left(\mathrm{LSD}_{0.05}\right)$ differences in productivity between $0 \%$ and $50 \%$ of the mean. (Bottom) The data of Fig. 9 (Top) plotted against the area occupied by the two experiments. It is intended to provide a rough comparison of the relative costs of the two experiments.

tree, was $\approx 75 \%$ above that found for standard peach trees on a per-unit-area basis (Hansche et al., 1985)]. These same data plotted on the area occupied by the two experiments are depicted in the bottom of Fig. 9. It is intended to provide a rough comparison of the relative costs of the two experiments.

The relevant point to be drawn from this comparison is that the experiment involving the dwarf trees was capable of discriminating differences of economic significance (8\% of the mean), and was practicable; i.e., within the range of resources available to most researchers of peach yield. Experiments of that power involving standard trees are beyond the resources of most researchers (Marini, 1985).

\section{CONCLUSIONS}

The direct relationship between seedling number and the power of genetic resolution was illustrated in the first section of this analysis. It can be seen from the analysis presented in the second section that seedling number has a less direct, but equally large, effect on the rate at which selection can increase the probability of desirable seedlings arising in breeding stocks. It was shown in the directly preceding section that dwarf peach trees facilitate a several-fold increase in the power of experiments designed to discriminate differences in genetic potential. Taken together, these results all demonstrate reduced tree size can facilitate a very large increase in the power of genetic analysis and manipulation in these species.

Reducing the length of the juvenile phase of these crops appears as straightforward as reducing their size (Hansche, 1986; Visser, 1965, 1972, 1976; Zielinski, 1963). The effect of its duration on genetic analysis and manipulation is direct. A short juvenile phase increases the rate of both processes by reducing the length of sexual cycles; consequently, its effect on selection progress is cumulative. Further, precocity and reduced plant size have a positive synergistic effect on the rate at which genetic manipulations can be effected. In short, smaller plants with short juvenile phases are attainable and can facilitate a large increase in our ability to analyze the products of meiosis and to construct desirable multigenic systems in fruit and nut tree species.

Recombinant DNA technology, whatever its contributions to genetic analysis and manipulation, is not expected to free the geneticist from the necessity of making crosses and of screening segregating progeny for the particular aggregates of multiple genes that characterize cultivars. That is to say, the technique does not promise to supersede the

the multiple gene systems of commercial cultivars. To the contrary, reductions in plant size and in the duration of the juvenile phase would accelerate the implementation of recombinant DNA technology in the genetic analysis of these species and in the development of superior cultivars. The point should not be lost, nor should its implication be underestimated, that it is in the plant species most amenable to genetic analysis and manipulation via classic Mendelian methods that the RFLP mapping technologies are being most avidly applied; e.g., Arabidopsis, tomato, potato, and maize.

The genetic modifications required to streamline Mendelian analysis and manipulation of fruit and nut tree species are in plain view. Effecting them requires no new, or even exotic, technology.

\section{Literature Cited}

Ali, M.A.M., S.O. Okiror, and D.C. Rasmusson. 1978. Performance of semi-dwarf barley. Crop Sci. 18:418-422.

Allard, R.W. 1961. Principles of plant breeding. Wiley, New York.

Beutel, J. and M. Gerdts. 1980. Univ. of California Kearney Agr. Center Annu. Rpt. 51.

Bauer, R. 1957. The induction of vegetative mutations in Ribes nigrum. Hereditas 43:323-337.

Dalrymple, D.G. 1980. Development and spread of semi-dwarf wheat and rice in the United States (an international perspective). USDA Econ. Rpt. 455.

Falconer, D.S. 1981. Introduction to quantitative genetics. 2nd ed. Longman, New York.

Hansche, P.E. and V. Beres 1980. Genetic remodeling of fruit and nut trees to facilitate cultivar improvement. HortScience 15:710-714.

Hansche, P.E., W. Beres, and R. Darnell. 1985. Yield potential of peach trees dwarfed by the dw gene. HortScience 21(6):1453-1455.

Hansche, P.E. 1986. Heritability of juvenility in peach. HortSciencc 21(5):1197-1198.

Hansche, P.E. 1989. Three brachytic dwarf peach cultivars: Valley Gem, Valley Red, and Valley Sun. HortScience 24:707-709.

Hooper, J. 1867. The Italian dwarf peach. Amer. J. Hort. 2:287-288.

Lammerts, W.E. 1945. The breeding of ornamental edible peaches for mild climates: I. Inheritance of tree and flower characters. Amer J. Bot. 32(2):53-61. 
McComb, A.J. and J.A. McComb. 1970. Growth substances and the relation between phenotype and genotype in Pisum sativum. Planta 91:235245.

Marini, R.J. 1985. Sample size estimates for peach tree growth and yield experiments. J. Amer. Soc. Hort. Sci. 110(5):604-608.

Mohr, H.C. 1962. Utilization of the genetic character for short internode in improvement of watermelon. Proc. Amer. Soc. Hort. Sci. 82:454459 .
Pleton, J.S. 1964. Genetic and morphogenetic studies of angiosperm single-gene dwarfs. Bot. Rev. 30:479-512.

Schertz, K.F., D.J. Rosenow, J.W. Johnson, and P.T. Gibson. 1974. Single $D_{\mathrm{w}_{3}}$ height effects in 4- and 3-dwarf hybrids of Sorghum bicolor L. Moench. Crop Sci. 14:875-877.

Stevenson, E.C. and M.L. Tomes. 1958. The commercial potential of the dwarf tomato. Proc. Amer. Soc. Hort. Sci. 72:385-389.

Visser, J. 1976. A comparison of apple and pear seedlings with reference to the juvenile period: II. Mode of inheritance. Euphytica 25:339-342. Visser, J. 1972. Environmental and genetic factors influencing the juvenile period in apple. Proc. Angers Fruit Breeding Symp. 1970. p. 101-116.

Visser, J. 1965. On the inheritance of the juvenile period in apple. Euphytica 14:125-134.

Zielinski, Q.B. 1963. Precocious flowering of pear seedlings carrying the "cardinal red" color gene. J. Hered. 54:75-78. 